\title{
Muir-Torre Syndrome Associated Periocular Sebaceous Neoplasms: Screening Patterns in the Literature and in Clinical Practice
}

\author{
Maya Eiger-Moscovich ${ }^{a}$ Ralph C. Eagle Jr. ${ }^{a}$ Carol L. Shields ${ }^{b}$ Hilary Racher $^{c}$ \\ Sara E. Lally ${ }^{b}$ Rona Z. Silkiss ${ }^{d}$ Jerry A. Shields ${ }^{b}$ Tatyana Milman ${ }^{a}$ e \\ ${ }^{a}$ Department of Ophthalmic Pathology, Wills Eye Hospital, Sidney Kimmel Medical College of Thomas Jefferson \\ University, Philadelphia, PA, USA; ${ }^{b}$ Ocular Oncology Service, Wills Eye Hospital, Sidney Kimmel Medical College \\ of Thomas Jefferson University, Philadelphia, PA, USA; ' Impact Genetics/Dynacare, Bowmanville, ON, Canada; \\ dDivision of Ophthalmic Plastic, Reconstructive and Orbital Surgery, California Pacific Medical Center, \\ San Francisco, CA, USA; ' Department of Pathology, Anatomy, and Cell Biology, Sidney Kimmel Medical College of \\ Thomas Jefferson University, Philadelphia, PA, USA
}

\section{Keywords}

Muir-Torre syndrome · Eyelid sebaceous neoplasm .

Screening patterns

\begin{abstract}
Background: Muir-Torre syndrome (MTS) is defined clinically as the association of cutaneous sebaceous neoplasm and visceral malignancy. Ancillary tests are considered crucial for diagnosis. Although screening guidelines for MTS, including the Mayo MTS scoring system, have been proposed, there are no ophthalmic site-specific guidelines. Summary: A literature review conducted by PubMed search for articles describing patients with periocular sebaceous neoplasm and MTS disclosed 31 publications describing 60 patients, 36 (60\%) of whom fulfilled clinical criteria for MTS, 6 (10\%) whose diagnosis was based on screening ancillary studies, $14(23 \%)$ who fulfilled clinical criteria and had supporting screening ancillary studies, and 4 (7\%) who fulfilled clinical criteria and had supporting diagnostic genetic testing. Most patients were male (34 vs. 15 females), with a median age of 59 years (range 37-79 years). The most common diagnosis was sebaceous carcinoma $(40 / 60,67 \%)$, followed by sebaceous adenoma $(16 / 60,27 \%)$, followed by other tumors with sebaceous differentiation $(4 / 60,6 \%)$. The periocular lesions
\end{abstract}

were identified prior to visceral malignancy in 10 out of 45 (22\%) cases, after visceral malignancy in 34 out of 45 (76\%) cases, and concurrently with visceral malignancy in 1 out of 45 (2\%) cases. Immunohistochemistry for mismatch repair proteins was performed in 41 out of $60(68 \%)$ and 14 out of $38(37 \%)$ of the tumors had lost MSH2. Based on Mayo-MTS scores of 2 or greater, and after removing visceral malignancies not included in their scoring algorithm, 26 out of 30 of patients $(87 \%)$ with complete data were considered to be appropriate candidates for further work-up. A survey of current practice was conducted by questionnaires, distributed to ophthalmic pathologists, ocular oncologists, and oculoplastic surgeons from national and international professional societies. Of the 103 physicians who participated in the survey, 91 (88\%) felt that MTS evaluation guidelines were not sufficiently clear. Key Messages: Our findings suggest that Mayo MTS screening guidelines may be applicable to periocular sebaceous neoplasms. The uncertainty of ophthalmic specialists about optimal screening guidelines for MTS reflects the heterogeneity of defining criteria for MTS and limited molecular genetic data. Larger studies with detailed clinical, histopathologic, and molecular genetic data are required to formally assess screening guidelines for MTS in patients with periocular sebaceous neoplasms.

(C) 2020 S. Karger AG, Base

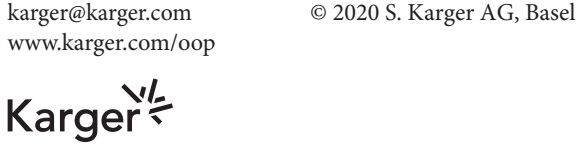

Tatyana Milman, MD

Department of Pathology, Wills Eye Hospital

840 Walnut Street

Philadelphia, PA 19107 (USA)

E-Mail tmilman@willseye.org 


\section{Introduction}

Sebaceous neoplasms include a spectrum of tumors ranging from sebaceous gland hyperplasia to sebaceous adenoma, epithelioma, and sebaceous carcinoma. Sebaceous neoplasms are most common in the periocular area, usually arising from Meibomian glands and less frequently from the sebaceous glands of Zeiss [1] and sebaceous glands in the caruncle.

Muir-Torre syndrome (MTS) is a rare autosomal-dominant disorder, defined by the association of cutaneous lesions (sebaceous neoplasms or keratoacanthoma) and visceral malignancies (mainly colorectal, endometrial, ovarian and renal pelvis/ureter carcinomas) in the absence of other predisposing factors [2]. MTS is a clinical variant of Lynch syndrome, constituting less than $5 \%$ of all cases. Lynch syndrome represents a hereditary nonpolyposis colorectal cancer and is the most common cause of inherited colorectal malignancy [3]. In a literature review of 205 MTS patients by Akhtar et al. [4], cutaneous sebaceous neoplasms at any site appeared before the visceral malignancy in $22 \%$, concurrently in $6 \%$, and following the visceral malignancy in $56 \%$ of cases. Although sebaceous neoplasms are most frequent in the periocular area, MTS is usually diagnosed in patients with non-periocular sebaceous neoplasms, not located in head and neck region [5-7].

The majority of patients with Lynch syndrome or MTS harbor germline pathogenic variants in DNA mismatch repair (MMR) genes: the mutL homolog1 (MLH1), mutS homolog2 (MSH2), mutS homolog6 (MSH6) and postmeiotic segregation increased2 (PMS2). In addition, germline deletions within the epithelial cell adhesion molecule gene have also been implicated in Lynch syndrome, as these deletions can disrupt the MMR pathway through the inactivation of the adjacent $\mathrm{MSH} 2$ gene [3]. The $4 \mathrm{MMR}$ genes encode $4 \mathrm{MMR}$ proteins that form complexes with each other in order to detect and repair errors in base pairing during DNA replication; MLH1 dimerizes with PMS2, while MSH2 dimerizes with MSH6 [8]. Germline alteration in one of the MMR genes followed by a second somatic hit to the remaining wild type allele [5] leads to genomic microsatellite instability (MSI) which, in turn, predisposes to the development of malignancies [2]. In Lynch syndrome-associated colorectal cancers, the loss of MLH1 protein is the most common MMR protein deficiency, followed by MSH2 loss. In contrast, the sebaceous neoplasms, including those associated with MTS, show loss of MSH2 expression in over $90 \%$ of cases, followed by loss of MLH1 $[5,6,9,10]$. An isolated deficiency of MSH6 or PMS2 has been documented [6].
The MMR gene pathogenic variants can be indirectly assessed in the tumor tissue using immunohistochemistry (IHC) evaluating MMR protein expression and by the polymerase chain reaction (PCR)-based MSI analysis. Interpretation of IHC for MMR proteins is complicated by the fact that MLH1 and MSH2 have multiple binding partners; while heterodimers are formed between MLH1 + PMS2 and MSH2 + MSH6, both MLH1 and MSH2 also form heterodimers with other MMR proteins. In contrast, PMS2 only binds to MLH1 and MSH6 only binds to MSH2. Immunohistochemically, this means that loss of MLH1 function will lead to loss of PMS2 staining and loss of MSH2 function will lead to loss of MSH6 staining, but not vice versa. Additionally, molecular genetic studies on the tumor and blood DNA can directly evaluate the MMR gene status [3]. In literature reviews by Zhang et al. [11] and Shia et al. [12], MMR IHC was found comparable to PCR-based MSI testing in Lynch syndrome-associated tumors. The authors concluded that the IHC panel evaluating expression of $4 \mathrm{MMR}$ proteins was rapid, cost-effective, sensitive, and highly specific, but could miss rare missense variants and, therefore, should be used as a screening method and interpreted cautiously. In most published studies on MTS, the authors used IHC MMR as a diagnostic, rather than screening method, applying it for evaluation of both cutaneous sebaceous neoplasms and visceral malignancies $[5,6,13,14]$. This traditional IHC-based diagnosis of MTS has been recently challenged by Roberts et al. $[9,15]$, who showed that the sensitivity and specificity of MMR IHC in predicting the underlying gene defect was 85 and $48 \%$, respectively, in sebaceous neoplasms with a false-positive rate of $56 \%$, compared to the sensitivity and specificity values of 92 94 and $88-100 \%$, respectively, in colorectal tumors. These observations led Roberts et al. $[9,15]$ to suggest that when tissue from the colon or endometrial cancer is not available for IHC analysis, a genetic testing for MTS should be undertaken.

Traditionally, the diagnosis of Lynch syndrome has been mainly clinical, based on various scoring systems, such as the Amsterdam II criteria, the revised Bethesda guidelines, and the colorectal cancer risk assessment tool. Several computational models including the MMRpredict, MMRpro, and PREMM models were developed in order to assist in the decision on when to perform molecular genetic testing in patients with colorectal carcinoma [3]. Currently, the final diagnosis of Lynch syndrome is made by molecular genetic testing, which separates Lynch syndrome (germline MMR pathogenic variant) from Lynch-like syndrome (biallelic somatic 
MMR pathogenic variant), familial colorectal cancer type $\mathrm{X}$ (no MSI and MMR pathogenic variant) and other subtypes [16]. According to the AJCC 8th edition, high levels of MSI occur in $15 \%$ of colorectal carcinomas. Testing for PCR-based MSI analysis is recommended either according to the established clinical and pathologic risk factors, or in all tumors, which makes MSI a level I factor to be collected [17].

Currently, there are no uniform diagnostic guidelines for MTS. Like Lynch syndrome, our approach to the diagnosis of MTS has evolved with the improved understanding of its underlying genetic basis. Until recently, the diagnosis of MTS was clinical, based on the combination of an MTS-associated cutaneous neoplasm, including sebaceous neoplasms, and a Lynch syndrome-related visceral malignancy, with or without a family history. With the advent of ancillary diagnostic studies, MMR IHC and PCR-based MSI analysis have emerged as useful screening studies for MTS, particularly in colorectal and endometrial tumors. Until recently, a conclusive diagnosis based on the identification of an underlying germline gene defect by molecular genetic testing has been seldom performed.

Recently, several groups attempted to establish screening guidelines for the evaluation of MTS in patients presenting with sebaceous neoplasms. In 2014, the Mayo MTS scoring system was developed by Roberts et al. [15], with a goal of establishing criteria associated with a higher risk of MTS in patients with sebaceous neoplasms. In that analysis, the diagnosis of MTS was established by MMR germline genetic testing. Roberts et al. [15] found that age younger than 60 , the presence of 2 or more sebaceous neoplasms, and a personal or family history of Lynch-related cancer are risk factors for MTS. The scores for these 4 risk factors were summed to create a total score, the "Mayo MTS risk score," with a possible range of $0-5$ (due to its high OR and individual predictive ability, the presence of 2 or more sebaceous neoplasms was assigned a score of 2, while the other 3 risk factors were assigned a score of 1; online suppl. Table 1; see www. karger.com/doi/10.1159/000504984 for all online suppl. material) [15]. The score of 2 or more had a sensitivity of $100 \%$ and specificity of $81 \%$ for predicting a germline pathogenic variant in a Lynch syndrome MMR gene. The authors recommended that every patient with a score 2 or higher be evaluated by MMR IHC on colon or endometrial cancer or, when not available, by molecular genetic testing. John et al. [18] offered an alternative screening algorithm, suggesting that any sebaceous neoplasm or multiple keratoacanthomas should prompt a discussion regarding a family and personal history of malignancy and MMR IHC. Deficient MMR protein expression in the tumor, according to John et al. [18], is an indication for MSI analysis, genetic counseling, and close cancer surveillance.

It should be noted that the proposed screening guidelines for MTS have been established for patients with sebaceous neoplasms irrespective of their location. Aside from the AJCC 8th edition, which suggested that the diagnosis of eyelid sebaceous neoplasm should prompt consideration of a workup to exclude MTS with level of evidence III [19], none of the available guidelines specifically addresses the screening for MTS in patients with periocular sebaceous neoplasms. Due to the relatively low association of periocular sebaceous neoplasm with MTS [5-7], the generalized MTS screening guidelines are possibly not applicable, and ophthalmic site-specific guidelines for MTS screening in patients with periocular sebaceous neoplasm may be required.

In order to assess whether periocular sebaceous neoplasms indeed necessitate separate screening guidelines, we performed a literature review focused on evaluating the frequency of association of periocular sebaceous neoplasm with MTS and on the diagnostic criteria used to establish the diagnosis. Further, using a questionnaire, we assessed the current practice patterns of ophthalmic pathologists, ocular oncologists, and oculoplastic surgeons regarding the evaluation of patients with periocular sebaceous neoplasm for MTS.

\section{Methods}

\section{Literature Review}

PubMed database was searched for articles containing the terms MTS AND eyelid or periocular or orbital. The search words screening, microsatellite instability (MSI), MMR, gene mutation, and IHC were added to each of the above search combinations. Additional articles found by citation search of the included articles were added to the list of publications. After removal of duplicate articles and articles not available in English, the full-text articles were assessed for eligibility by excluding publications that (1) did not provide specific information on the diagnostic criteria for MTS; (2) had insufficient or absent information on the periocular sebaceous neoplasm, or (3) described only patients with extraocular sebaceous neoplasm (Fig. 1).

The patients described in the articles selected for review were subdivided in 5 groups based on how the diagnosis of MTS was established, based on clinical diagnostic criteria (periocular sebaceous neoplasm with/without MTS-associated visceral malignancy) and ancillary studies (IHC, MSI, or molecular genetic testing) as follows:

1. Group 1 - Clinical diagnostic criteria (periocular sebaceous neoplasm with MTS-associated visceral malignancy) without ancillary studies, or patients fulfilling clinical diagnostic criteria with MMR IHC and MSI analysis not supportive of MTS. 


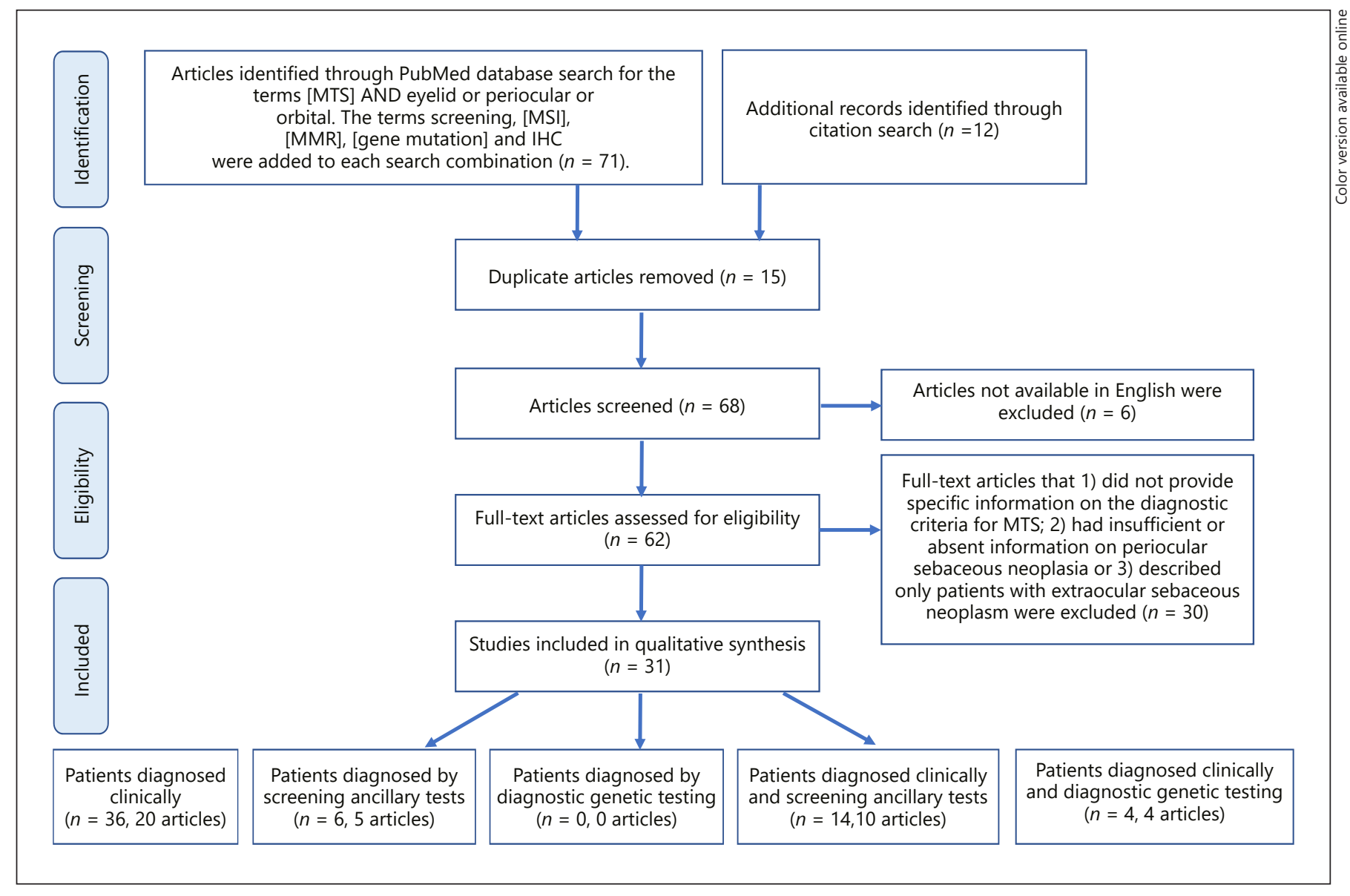

Fig. 1. Prisma flowchart of the systematic review process for selection of articles to be included in qualitative analysis of cases of MTS with periocular sebaceous neoplasm.

2. Group 2 - Clinical periocular sebaceous neoplasm (without documentation of MTS-associated visceral malignancy) and with supporting screening ancillary studies (IHC or MSI).

3. Group 3 - Clinical periocular sebaceous neoplasm (without documentation of MTS-associated visceral malignancy) and with supporting diagnostic molecular genetic testing.

4. Group 4 - Clinical diagnostic criteria (periocular sebaceous neoplasm with MTS-associated visceral malignancy) and with supporting screening ancillary studies (IHC or MSI).

5. Group 5 - Clinical diagnostic criteria (periocular sebaceous neoplasm with MTS-associated visceral malignancy) and with supporting diagnostic molecular genetic testing.

\section{Survey of Current Practice}

Ophthalmic pathologists, ocular oncologists, and oculoplastic surgeons, members of the International Society of Ocular Oncology, Eastern Ophthalmic Pathology Society, American Association of Ophthalmic Oncologists and Pathologists and the American Society of Ophthalmic Plastic and Reconstructive Surgery were asked to describe their screening patterns for MTS using an Internet survey. The surveys are included as supplementary material (online suppl. Files 1-3).

Periocular MTS Screening Patterns
The Institutional Review Board deemed that this study was exempt from IRB approval. The study adhered to the tenets of the declaration of Helsinki.

\section{Results}

\section{Literature Review}

The PubMed database search revealed 71 articles. To that we added 12 articles identified through citation search. After exclusion of duplicate articles, non-English written articles and non-relevant articles (as specified in the methods section) 31 articles were included in the qualitative synthesis (Fig. 1). The articles were classified with criteria for group 1 (clinical diagnostic criteria of periocular sebaceous neoplasm with MTS-associated visceral malignancy without/with negative ancillary studies), comprising 20 published papers describing 36 patients [13, 14, 20-37], group 2 (periocular sebaceous neo- 
plasm with supporting screening ancillary studies [IHC or MSI] without documentation of MTS-associated visceral malignancy), comprising 5 published papers describing 6 patients $[5,14,31,36,38]$, group 3 (periocular sebaceous neoplasm with supporting diagnostic molecular genetic testing without documentation of MTS-associated visceral malignancy), comprising zero articles, group 4 (clinical diagnostic criteria of periocular sebaceous neoplasm with MTS-associated visceral malignancy with supporting screening ancillary studies), comprising 10 published papers describing 14 patients $[8,14,26$, $29,32,39-43$ ] and group 5 (clinical diagnostic criteria of periocular sebaceous neoplasm with MTS-associated visceral malignancy with supporting diagnostic molecular genetic testing) comprising 4 published papers describing 4 patients $[41,44-46]$. There were 7 articles describing 31 patients who were stratified into several groups $[13,26$, 29, 31, 32, 36, 41] (Table 1).

The demographic information was available on 49 of 60 identified patients $[5,8,13,14,20-46]$. The patients were predominantly male ( 34 male vs. 15 females, $2.3: 1$ ), at a median age of 59 years (range 37-79). Twenty two of 40 patients (55\%) had documented the family history of visceral malignancies in first- and second-degree relatives. The most common cutaneous diagnoses included sebaceous carcinoma (40/60,67\%), sebaceous adenoma $(16 / 60,27 \%)$, keratoacanthoma (2/60,3\%), sebaceous epithelioma $(1 / 60,1.5 \%)$, and squamous cell carcinoma with sebaceous differentiation (1/60,1.5\%). The degree of differentiation was recorded in 5 of 40 sebaceous carcinomas as well-differentiated in 4 out of $5(80 \%)$ and poorly differentiated in 1 out of $5(20 \%)$ cases. The information on tumor differentiation was not documented in the remaining 35 sebaceous carcinomas. Of 28 sebaceous neoplasms with documented location, the location included upper eyelid $(18 / 27,67 \%)$, lower eyelid $(8 / 27,29 \%)$ or medial canthus $(1 / 27,4 \%)$. In 44 cases where the number of sebaceous neoplasms was documented, 33 patients $(33 / 44,75 \%)$ had a single sebaceous neoplasm, while 11 patients $(11 / 44,25 \%)$ had multiple sebaceous neoplasms, which could be temporally, spatially, and diagnostically distinct. Visceral malignancy was documented in 54 of 60 (94\%) patients. Of 45 patients with a specified timeline, 10 of 45 sebaceous neoplasms (22\%) were diagnosed prior to the visceral malignancy, 1 sebaceous neoplasm (2\%) was diagnosed concurrently with the visceral malignancy, and most sebaceous neoplasms (34/45, 76\%) were identified following the diagnosis of visceral malignancy. The most common ancillary study was an immunohistochemical panel for MMR protein expression, performed on 41 of $60(68 \%)$ tumors. Fifteen of $41(37 \%)$ tumors were evaluated with a panel of 4 antibodies (MSH2, MSH6, MLH1, and PMS2), 8 out of 41 (20\%) tumors with a panel of 3 antibodies (MSH2, MLH1, and MSH6), 9 out of $41(22 \%)$ tumors with a panel of 2 antibodies (MSH2 and MLH1), and 7 out of 41 (17\%) tumors with one antibody (MSH2; Table 1). The most common deficient protein was MSH2 in 14 out of 38 tumors (37\%), followed by MLH1 in 6 out of 31 (19\%), PMS2 in 2 out of $13(15 \%)$ and MSH6 in 3 out of 22 (14\%). In 3 tumors, the deficient protein was not specified (Table 1). Fourteen of 60 tumors (23\%) were tested for MSI, which was unstable in 6 out of $14(43 \%)$ lesions, and abnormal but not diagnostic in 1 out of 14 (7\%). All 7 tumors with MSI also demonstrated MMR protein expression loss (100\%). Similarly, all 7 microsatellite stable tumors evaluated by MMR IHC had retained MMR protein expression (100\%). Four of 60 patients (7\%, group 5), 2 males (50\%) and 2 females ( $50 \%$, $1: 1$ ), with a median age of 55 (range 46-65) underwent diagnostic molecular genetic testing, which demonstrated a germline MMR gene defect in all 4 (100\%), hMSH2 in $3(75 \%)$ and hMLH1 in $1(25 \%)$ patient. Two patients (50\%) had documented family history of visceral malignancies in first- and second-degree relatives. Three patients had sebaceous carcinoma (75\%), which were multiple in 2 out of 3,67\%. Differentiation was not specified in sebaceous carcinomas. The remaining 1 patient (25\%) had a single sebaceous adenoma. All 4 patients had visceral malignancy diagnosed prior to the sebaceous neoplasm. The patient with $h M L H 1$ pathogenic variant had concurrent MLH1 protein expression loss. MMR IHC studies and MSI analysis were not performed on the tumors of 3 other patients. There was no appreciable difference in the demographic and clinical parameters of the patients in the 5 groups (Table 1). Per our inclusion criteria, the ancillary studies were normal in all evaluated tumors from the patients in group 1. The MMR protein IHC and MSI analysis results were similar between groups 2 and 4 tumors.

Applying the Mayo MTS scoring system to the 60 identified patients, 35 out of 60 patients (58\%) had a score of 2 or greater, making them appropriate candidates for further workup according to the Mayo MTS scoring system. When adjusting this analysis to include only those patients with complete information on all 4 risk factors, 28 out of 30 patients $(93 \%)$ had a score of 2 or greater. When adjusting our analysis to include only the MTSassociated visceral malignancies as defined in the algorithm by Roberts et al. [15], namely, colorectal, endometrial, ovarian, small bowel, urinary tract, and biliary tract 


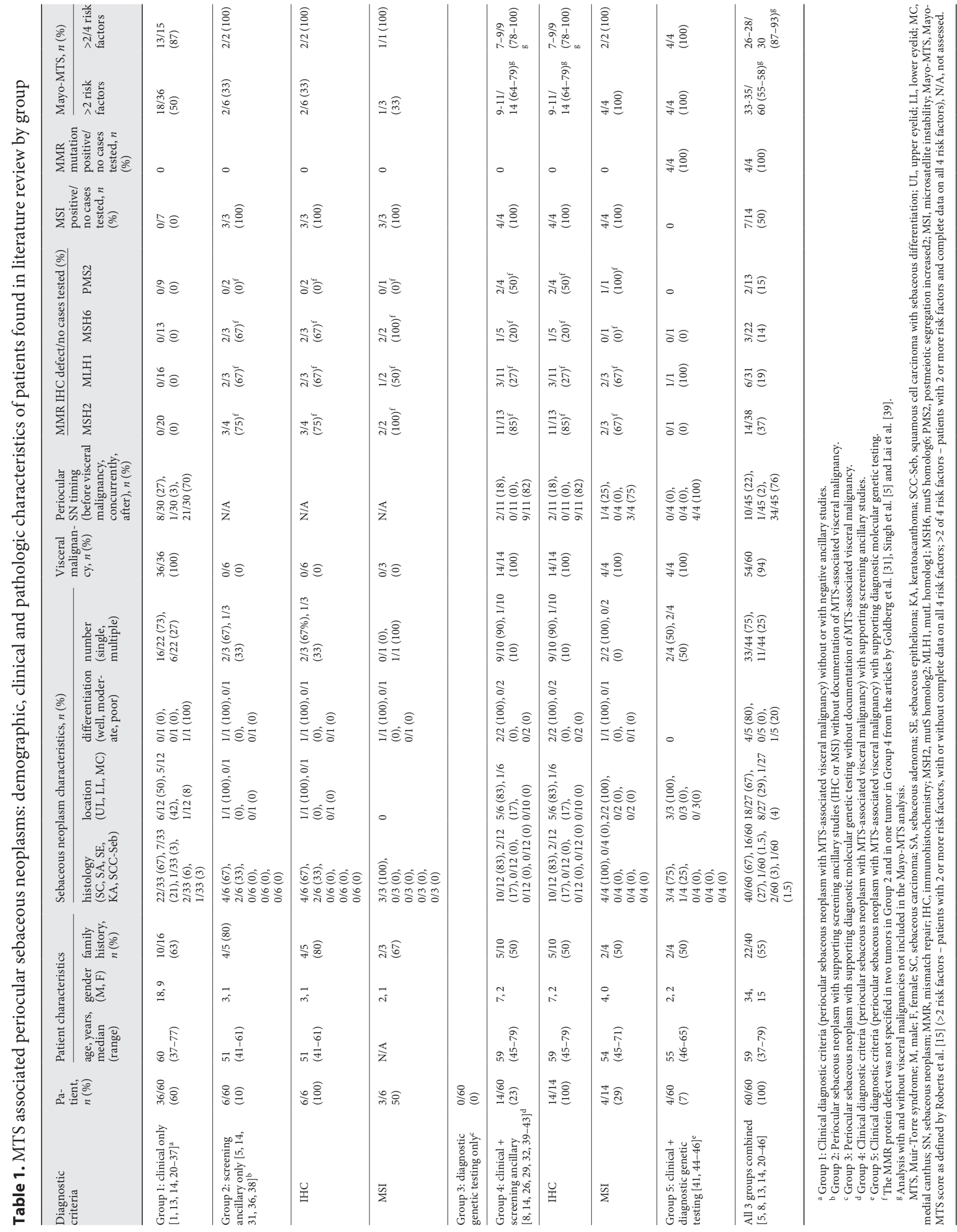


Table 2. MTS-associated periocular sebaceous neoplasms: survey responders

\begin{tabular}{lccc}
\hline & $\begin{array}{l}\text { Ophthalmic pathologists, } \\
n(\%)\end{array}$ & $\begin{array}{l}\text { Ocular oncologists, } \\
n(\%)\end{array}$ & $\begin{array}{l}\text { Oculoplastic surgeons, } \\
n(\%)\end{array}$ \\
\hline Practice location & & & \\
USA & $28 / 56(50)$ & $8 / 31(26)$ & $13 / 16(81)$ \\
Europe & $11 / 56(20)$ & $3 / 31(10)$ & $0 / 16(0)$ \\
Canada & $2 / 56(3.5)$ & $2 / 31(6)$ & $1 / 16(6)$ \\
Asia & $6 / 56(11)$ & $8 / 31(26)$ & $0 / 16(0)$ \\
Australia & $3 / 56(5)$ & $3 / 31(10)$ & $1 / 16(6)$ \\
Central/South America & $4 / 56(7)$ & $5 / 31(16)$ & $1 / 16(6)$ \\
Other & $2 / 56(3.5)$ & $2 / 31(6)$ & $0 / 16(0)$ \\
Practice type & & & $1 / 16(6)$ \\
Eye pathology/ & $39 / 56(70)$ & $20 / 31(65)$ & $3 / 16(19)$ \\
Ophthalmology hospital & & & $12 / 16(75)$ \\
General pathology/ & $10 / 56(18)$ & $5 / 31(16)$ & $0 / 16(0)$ \\
Oncology/plastics hospital & $2 / 56(3)$ & $4 / 31(13)$ & $2 / 31(6)$ \\
Private practice & $5 / 56(9)$ & $4 / 31(13)$ & $2 / 16(12)$ \\
Other & $6 / 55(11)$ & $8 / 31(26)$ & $6 / 16(38)$ \\
Are MTS screening guidelines for patients with periocular sebaceous neoplasia clear? & $6 / 16(38)$ \\
Extremely clear & $23 / 55(42)$ & $3 / 31(51)$ & $2 / 16(12)$ \\
Somewhat clear & $18 / 55(33)$ & & \\
Not so clear & $8 / 55(14)$ & & \\
Not at all clear & & & \\
\hline
\end{tabular}

MTS, Muir-Torre Syndrome.

cancers, 33 of 60 patients (55\%) had a score of 2 or greater and 26 of 30 patients (87\%) with complete information on all 4 risk factors had a score of 2 or greater (online suppl. Table 2).

\section{Survey of Current Practice}

The questionnaire was distributed to 70 ophthalmic pathologists using personalized email, 290 ocular oncologists using non-personalized email, and 860 oculoplastic surgeons using a website advertisement.

There were 103 physicians who answered our Internet survey, including 56 ophthalmic pathologists (56/70, $80 \%), 31$ ocular oncologists $(31 / 290,11 \%)$ and 16 oculoplastic surgeons $(16 / 860,2 \%)$. Of the responders, the majority of ophthalmic pathologists and oculoplastic surgeons practice in the United States (50 and 81\%, respectively), while the ocular oncologists were more widely distributed geographically (USA 26\%, Asia 26\%; Table 2). The majority of ophthalmic pathologists and ocular oncologists practice in an eye pathology or ophthalmology department in a hospital or academic center (70 and 65\% respectively), while oculoplastic surgeons predominantly work at a private practice $(75 \%)$. The majority of responders from the 3 subspecialties stated that the guidelines for
MTS evaluation in patients with periocular sebaceous neoplasm were unclear ( $89 \%$ of ophthalmic pathologists, $87 \%$ of ocular oncologists, and $88 \%$ of oculoplastic surgeons; Table 2).

The questionnaire of the ophthalmic pathologists revealed that the majority $(38 / 56,68 \%)$ use MMR IHC to exclude MTS. MMR IHC usually was performed on sebaceous adenomas and epitheliomas $(15 / 38,39 \%)$, well-differentiated sebaceous carcinomas $(12 / 38,32 \%)$ and/or on tumors of patients with personal or family history concerning for MTS (15/38, 39\%; online suppl. Table 3, Fig. 2). Of 40 ophthalmic pathologists who specified the immunohistochemical stains of choice, 30 pathologists (75\%) used a panel of 4 antibodies, 2 out of 40 pathologists (5\%) used a panel of 3 antibodies (MSH2, MLH1 and MSH6 or PMS2), 5 out of 40 pathologists (13\%) used a panel of 2 antibodies (MSH2 and MLH1 or MSH6), and 3 out of 40 pathologists (7\%) used 1 antibody (MSH2 or MSH6). Of the 18 ophthalmic pathologists $(18 / 56,32 \%)$ who do not use MMR IHC, the majority $(12 / 18,67 \%)$ did not have access to MMR IHC, either in their laboratory or as a send out test (online suppl. Table 3, Fig. 2). Only $43 \%(24 / 56)$ of responders use MSI analysis and $38 \%$ of responders (21/56) used MMR genetic testing (online 


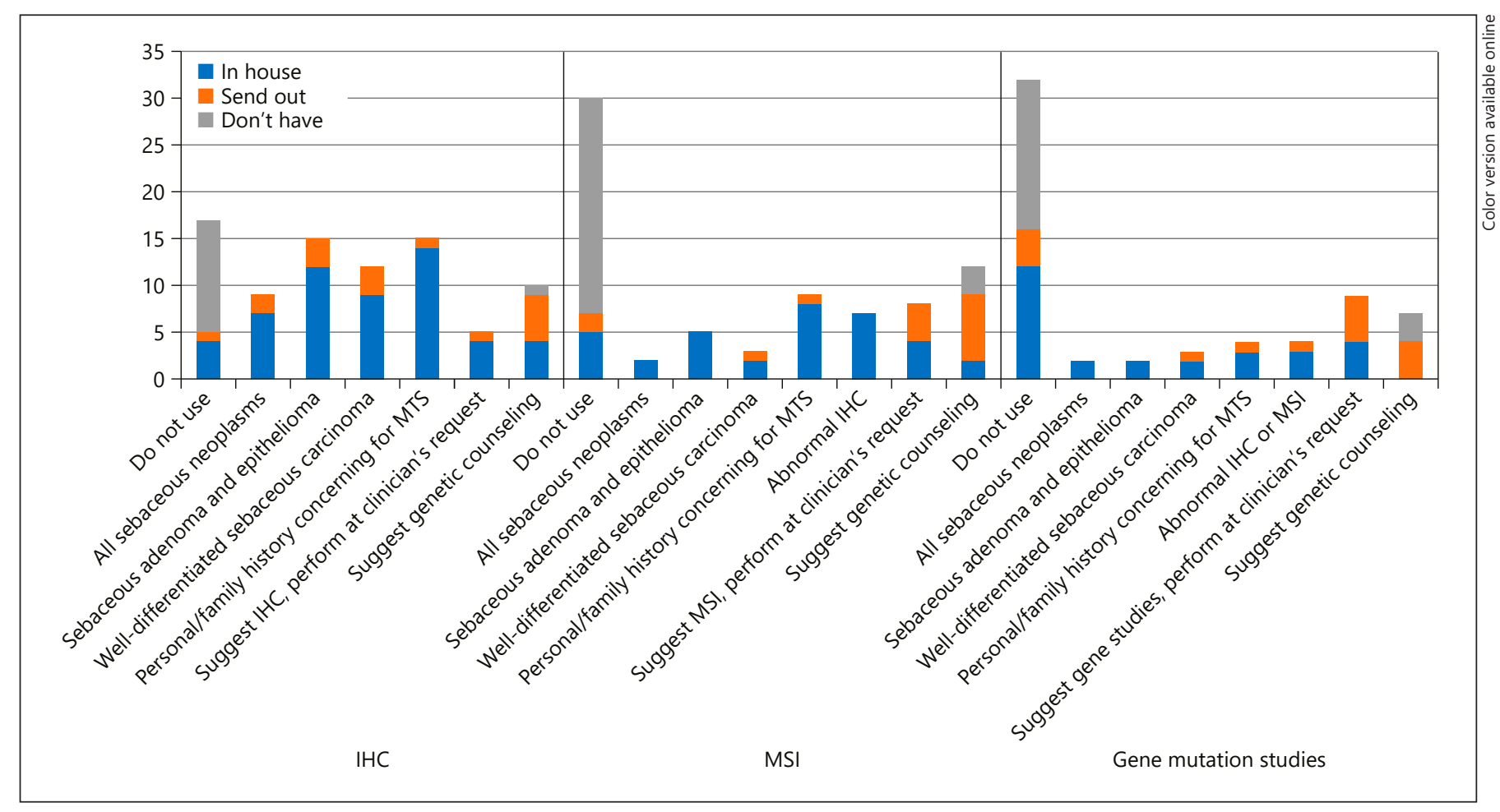

Fig. 2. Ophthalmic pathologists' usage of IHC, MSI analysis and genetic testing for the screening of MTS. IHC, immunohistochemistry; MSI, microsatellite instability.

suppl. Table 3, Fig. 2). Twenty-three of 32 (72\%) of responders who did not perform MSI did not have access to this test, either in their laboratory or as a send-out test. Similarly, 16 of 35 (46\%) responders who did not perform MMR genetic testing did not have access to this test (online suppl. Table 3, Fig. 2). Fifteen of 56 (27\%) ophthalmic pathologists included recommendations on genetic counseling in their report.

The questionnaires completed by ocular oncologists revealed that the leading indications for MTS work-up were the presence of an MTS-associated visceral neoplasm $(17 / 31,55 \%)$ and/or the pathologist's recommendation (13/31, 42\%; Table 3). Most ocular oncologists referred the patient to a non-ocular oncologist for systemic evaluation (18/31, 58\%; Table 3). Most ocular oncologists deferred to the pathologist for recommendations on obtaining ancillary MMR IHC, MSI, and genetic testing (48, 58 , and $61 \%$ respectively; Table 3 ).

The questionnaires completed by the oculoplastic surgeons, although likely biased due to low response rate, revealed that the leading indication for MTS work-up was the diagnosis of a sebaceous neoplasm (7/16, 44\%; Table 3). Most oculoplastic surgeons referred the patient to a non-ocular oncologist for systemic evaluation $(8 / 14,57 \%$; Table 3). Most oculoplastic surgeons deferred to the pathologist for recommendations on obtaining ancillary MMR IHC, MSI, and genetic testing (50, 69, and 75\% respectively; Table 3 ).

\section{Discussion}

MTS is a rare disorder, usually defined clinically, by the association of a cutaneous sebaceous neoplasm and visceral malignancy in the absence of other predisposing factors [2]. In recent years, ancillary immunohistochemical and molecular genetic studies have become increasingly important in the diagnosis of MTS. However, there are no uniformly accepted guidelines for MTS screening $[15,18]$ and no specific guidelines for MTS screening of patients with periocular sebaceous neoplasia.

The absence of ophthalmic site-specific guidelines contributes to management challenges of patients with periocular sebaceous neoplasms. Although it is well recognized that sebaceous neoplasms occur most frequently in the periocular area, most sebaceous tumors associated 
Table 3. Ocular oncologists and oculoplastic surgeons screening recommendations for MTS

\begin{tabular}{|c|c|c|}
\hline & Ocular oncologists, $n(\%)$ & Oculoplastic surgeons, $n(\%)$ \\
\hline \multicolumn{3}{|l|}{ What prompts evaluation for MTS? } \\
\hline Do not evaluate & $5 / 31(16)$ & $5 / 16(32)$ \\
\hline All sebaceous neoplasms & $6 / 31(19)$ & $7 / 16(44)$ \\
\hline Amsterdam II criteria & $3 / 31(10)$ & $0 / 16(0)$ \\
\hline Revised Bethesda guidelines & $1 / 31(3)$ & $1 / 16(6)$ \\
\hline Visceral neoplasm & $17 / 31(55)$ & $3 / 16(19)$ \\
\hline Well-differentiated sebaceous neoplasms & $6 / 31(19)$ & $2 / 16(13)$ \\
\hline Sebaceous neoplasm with abnormal IHC/MSI & $5 / 31(16)$ & $3 / 16(19)$ \\
\hline Pathologist recommendation & $13 / 31(42)$ & $4 / 16(25)$ \\
\hline Young age & $4 / 31(13)$ & $1 / 16(6)$ \\
\hline \multicolumn{3}{|l|}{ How do you evaluate for MTS? } \\
\hline Non-ocular oncologists & $18 / 31(58)$ & $8 / 14(57)$ \\
\hline Geneticist & $9 / 31(29)$ & $1 / 14(7)$ \\
\hline Gastroenterologist & $8 / 31(26)$ & $4 / 14(29)$ \\
\hline $\mathrm{PET} / \mathrm{CT}$ & $8 / 31(26)$ & $0 / 14(0)$ \\
\hline Pathologist & $11 / 31(35)$ & $4 / 14(29)$ \\
\hline Other & $2 / 31(6)$ & $3 / 14(21)$ \\
\hline \multicolumn{3}{|l|}{ How do you use IHC? } \\
\hline Pathologist's discretion & $15 / 31(48)$ & $8 / 16(50)$ \\
\hline All sebaceous neoplasms & $5 / 31(16)$ & $4 / 16(25)$ \\
\hline Well-differentiated sebaceous neoplasms & $7 / 31(23)$ & $1 / 16(6)$ \\
\hline Personal/family history concerning for MTS & $9 / 31(29)$ & $3 / 16(19)$ \\
\hline Other & $4 / 31(13)$ & $1 / 16(6)$ \\
\hline \multicolumn{3}{|l|}{ How do you use MSI? } \\
\hline Pathologist's discretion & $18 / 31(58)$ & $11 / 16(69)$ \\
\hline All sebaceous neoplasms & $2 / 31(6)$ & $2 / 16(13)$ \\
\hline Well-differentiated sebaceous neoplasms & $5 / 31(16)$ & $0 / 16(0)$ \\
\hline Abnormal IHC & $4 / 31(13)$ & $0 / 16(0)$ \\
\hline Personal/family history concerning for MTS & $6 / 31(19)$ & $0 / 16(0)$ \\
\hline Geneticist's recommendation & $4 / 31(13)$ & $1 / 16(6)$ \\
\hline Other & $4 / 31(13)$ & $2 / 16(13)$ \\
\hline \multicolumn{3}{|l|}{ How do you use gene mutation studies? } \\
\hline Pathologist's discretion & $19 / 31(61)$ & $12 / 16(75)$ \\
\hline All sebaceous neoplasms & $1 / 31(3)$ & $1 / 16(6)$ \\
\hline Well-differentiated sebaceous neoplasms & $3 / 31(10)$ & $0 / 16(0)$ \\
\hline Abnormal IHC/MSI & $6 / 31(19)$ & $0 / 16(0)$ \\
\hline Personal/family history concerning for MTS & $5 / 31(16)$ & $0 / 16(0)$ \\
\hline Geneticist's recommendation & $6 / 31(19)$ & $1 / 16(6)$ \\
\hline Other & $4 / 31(13)$ & $2 / 16(13)$ \\
\hline
\end{tabular}

MTS, Muir-Torre syndrome; IHC, immunohistochemistry; MSI, microsatellite instability.

with MTS are in non-periocular skin [5-7, 47]. Singh et al. [5] documented this phenomenon in their study of 94 sebaceous lesions and found that MMR IHC deficiency was significantly associated with age (younger than 50 years), anatomic location (non-head and neck, $p<0.0001$ and non-periocular, $p=0.0127$ ), tumor type (head and neck adenoma), and architecture (keratoacanthomalike). Jessup et al. [6] similarly evaluated 216 sebaceous lesions with MMR IHC and found an association of MMR deficiency with anatomic location (non-head and neck), although the authors observed no association with patient age or tumor type. Rajan et al. [36] evaluated 11 eyelid sebaceous carcinomas by IHC, 7 of which also underwent MSI analysis, and found only 1 tumor with an MMR IHC deficiency and abnormal but non-diagnostic MSI, in a patient with a known family history of MTS. Finally, more recent molecular genetic studies of sebaceous neoplasms documented a different mutational profile in peri- 
ocular sebaceous carcinoma (TP53, RB1, and FHIT) when compared to its non-periocular counterpart $[1,31]$. The combined evidence suggests that a site-specific approach to MTS screening in patients with periocular sebaceous neoplasms should be considered.

Our literature review of 60 patients with periocular sebaceous neoplasms and MTS, disclosed no significant difference in the demographic and clinical characteristics of patients diagnosed solely by MTS clinical criteria and those diagnosed by clinical criteria in conjunction with supporting ancillary studies. The patients with periocular sebaceous neoplasms and MTS were predominantly male whose average age at the time of diagnosis of sebaceous neoplasm was younger than 60 years, and who had a personal and/or family history of MTS-related visceral malignancy - characteristics similar to those reported in patients with non-periocular sebaceous neoplasms associated with MTS $[15,18,29]$. Applying the Mayo MTS scoring system, and after removing visceral malignancies not included in their analysis, 33 out of 60 patients (55\%) would be candidates for further workup, when adjusted to include patients with complete risk factor data, 26 out of $30(87 \%)$ patients would be candidates for further workup. These findings suggest that the established clinical risk factors and the Mayo MTS scoring system may be adapted to the initial MTS screening guidelines of patients with periocular sebaceous neoplasms.

From the standpoint of MTS-associated periocular sebaceous neoplasms, $37-41 \%$ of all tumors with aberrant MMR protein expression demonstrated the loss of MSH2 protein expression with corresponding MSI. These data differ from the quoted $90 \%$ rate of $\mathrm{MSH} 2$ loss in prior studies of MTS-associated sebaceous tumors irrespective of site $[5,6]$, potentially reflecting the underlying molecular genetic differences between the periocular and nonperiocular sebaceous neoplasms. Unexpectedly, $67 \%$ of MTS-associated periocular neoplasms in the 60 patients in the literature were sebaceous carcinomas, rather than sebaceous adenomas and epitheliomas. This contrasts with several prior studies documenting a higher frequency of MTS in well-differentiated sebaceous neoplasms [5, $6,8,13,14,27,36,47]$. This discrepancy could be explained by a selection bias (only patients with sebaceous carcinoma were included in some studies [14, 26, 31]), by a lack of discrimination between sebaceous carcinoma and well-differentiated sebaceous neoplasms, and by the absence of uniform diagnostic criteria for MTS.

Notably, only 4 of 60 reported patients underwent germline MMR molecular genetic analysis. In light of our improved understanding of the molecular genetic basis of
MTS and considering recognized limitations of clinical models and immunohistochemical diagnostic studies, this paucity of molecular genetic information brings into question our interpretation of the published data. Studies similar to the 1 by Roberts et al. [15], based on the integrated clinical and molecular genetic diagnosis of MTS, are needed to establish site-specific MTS screening criteria for patients with periocular sebaceous neoplasms. This is particularly important, given the recently recognized molecular genetic differences between the periocular and non-periocular sebaceous neoplasms $[1,31]$.

From the survey of current practice patterns, we learned that $88 \%$ of ophthalmic pathologists, ocular oncologists, and oculoplastic surgeons feel that the guidelines for evaluation for MTS in patients with periocular sebaceous neoplasm are not sufficiently clear. A comparison of dermatopathologists' [2] and ophthalmic pathologists' responses to questionnaires regarding utilization of MMR IHC on sebaceous neoplasms (online suppl. Table 4) reveals that ophthalmic pathologists are less likely to utilize this test reflexively on all sebaceous neoplasms $(9 / 55,16$ vs. $38 / 133,29 \%)$ and to recommend and perform it at clinician's request $(5 / 55,9$ vs. $83 / 133,62 \%)$. The decisions that ophthalmic pathologists make regarding MMR IHC appear to be greatly influenced by (1) the neoplasm type (well-differentiated tumor) and (2) family/ personal history of MTS-related cancer. These differences in the MMR IHC utilization may reflect recognition by the ophthalmic pathologists of site-specific features of periocular sebaceous neoplasms. Additionally, the observation that 13 out of $56(23 \%)$ ophthalmic pathologists do not have access to MMR IHC in comparison to 6 out of 141 (4\%) dermatopathologists who may reflect the differences between dermatopathology and ophthalmic pathology laboratories. Most ophthalmic pathologists do not use MSI or MMR genetic testing, generally because these studies are either not readily available or cost-prohibitive. Ocular oncologists tend to evaluate for MTS in patients who have MTS-associated visceral neoplasm or at pathologist's recommendation. Our findings regarding oculoplastic surgeons may be biased since only $2 \%$ of American Society of Ophthalmic Plastic and Reconstructive Surgery members responded to the questionnaire. Nevertheless, it was notable that most respondents evaluated every patient with sebaceous neoplasm for MTS. Both ocular oncologists and oculoplastic surgeons refer the patients with suspected MTS to non-ocular oncologists for systemic oncologic evaluation and defer to the pathologist to perform ancillary diagnostic studies on tissue. 


\section{Conclusions}

Our study highlights the marked heterogeneity of the diagnostic criteria for MTS in patients with periocular sebaceous neoplasm and extremely limited supporting molecular genetic data, making the diagnosis of most patients in the published literature debatable. The lack of clear ophthalmic site-specific guidelines for MTS screening in patients with periocular sebaceous neoplasm is reflected in the responses to the questionnaires by ophthalmic pathologists, ocular oncologists, and oculoplastic surgeons. These findings suggest that although the Mayo-MTS screening guidelines may be applicable to periocular sebaceous neoplasms, collaborative large studies, documenting clinical and histopathological features and molecular genetic data on patients with periocular sebaceous neoplasms are required to delineate optimal MTS screening criteria.

\section{Acknowledgment}

The authors acknowledge Jaime Jessen (Impact Genetics/Dynacare) for her help in coordination of this study.

\section{Statement of Ethics}

The Institutional Review Board deemed that this study was exempted from IRB approval. The study adhered to the tenets of the declaration of Helsinki.

\section{Disclosure Statement}

The authors have no conflict of interest to disclose.

\section{Funding Sources}

This work was supported by the Eye Pathology Research and Education Fund.

\section{Author Contributions}

M.E.-M., R.C.E., C.L.S., S.E.L., R.Z.S., J.A.S., and T.M.: data collection, critical analysis of data and editing of the manuscript. M.E.-M.: drafting the manuscript. H.R.: critical analysis of data and editing of the manuscript. T.M.: technical and financial support.

\section{References}

1 Tetzlaff MT, Singh RR, Seviour EG, Curry JL, Hudgens CW, Bell D, et al. Next-generation sequencing identifies high frequency of mutations in potentially clinically actionable genes in sebaceous carcinoma. J Pathol. 2016 Sep; 240(1):84-95.

2 Lee JB, Litzner BR, Vidal CI. Review of the current medical literature and assessment of current utilization patterns regarding mismatch repair protein immunohistochemistry in cutaneous Muir-Torre syndrome-associated neoplasms. J Cutan Pathol. 2017 Nov; 44(11):931-7.

3 Giardiello FM, Allen JI, Axilbund JE, Boland CR, Burke CA, Burt RW, et al.; US Multi-Society Task Force on Colorectal Cancer. Guidelines on genetic evaluation and management of Lynch syndrome: a consensus statement by the US Multi-Society Task Force on colorectal cancer. Gastroenterology. 2014 Aug;147(2): $502-26$.

4 Akhtar S, Oza KK, Khan SA, Wright J. MuirTorre syndrome: case report of a patient with concurrent jejunal and ureteral cancer and a review of the literature. J Am Acad Dermatol. 1999 Nov;41(5 Pt 1):681-6.

5 Singh RS, Grayson W, Redston M, Diwan AH, Warneke CL, McKee PH, et al. Site and tumor type predicts DNA mismatch repair status in cutaneous sebaceous neoplasia. Am J Surg Pathol. 2008 Jun;32(6):936-42.
6 Jessup CJ, Redston M, Tilton E, Reimann JD. Importance of universal mismatch repair protein immunohistochemistry in patients with sebaceous neoplasia as an initial screening tool for Muir-Torre syndrome. Hum Pathol. 2016 Mar;49:1-9.

7 Dores GM, Curtis RE, Toro JR, Devesa SS, Fraumeni JF Jr. Incidence of cutaneous sebaceous carcinoma and risk of associated neoplasms: insight into Muir-Torre syndrome. Cancer. 2008 Dec;113(12):3372-81.

8 Jagan L, Zoroquiain P, Bravo-Filho V, Logan P, Qutub M, Burnier MN Jr. Sebaceous adenomas of the eyelid and Muir-Torre Syndrome. Br J Ophthalmol. 2015 Jul;99(7):90913.

9 Roberts ME, Riegert-Johnson DL, Thomas BC, Thomas CS, Heckman MG, Krishna M, et al. Screening for Muir-Torre syndrome using mismatch repair protein immunohistochemistry of sebaceous neoplasms. J Genet Couns. 2013 Jun;22(3):393-405.

10 Everett JN, Raymond VM, Dandapani M, Marvin M, Kohlmann W, Chittenden A, et al. Screening for germline mismatch repair mutations following diagnosis of sebaceous neoplasm. JAMA Dermatol. 2014 Dec;150(12): 1315-21.

11 Zhang X, Li J. Era of universal testing of microsatellite instability in colorectal cancer. World J Gastrointest Oncol. 2013 Feb;5(2): 12-9.
12 Shia J. Immunohistochemistry versus microsatellite instability testing for screening colorectal cancer patients at risk for hereditary nonpolyposis colorectal cancer syndrome. Part I. The utility of immunohistochemistry. J Mol Diagn. 2008 Jul;10(4):293300 .

13 Liau JY, Liao SL, Hsiao CH, Lin MC, Chang HC, Kuo KT. Hypermethylation of the CD$\mathrm{KN} 2 \mathrm{~A}$ gene promoter is a frequent epigenetic change in periocular sebaceous carcinoma and is associated with younger patient age. Hum Pathol. 2014 Mar;45(3):533-9.

14 Gaskin BJ, Fernando BS, Sullivan CA, Whitehead K, Sullivan TJ. The significance of DNA mismatch repair genes in the diagnosis and management of periocular sebaceous cell carcinoma and Muir-Torre syndrome. Br J Ophthalmol. 2011 Dec;95(12):1686-90.

15 Roberts ME, Riegert-Johnson DL, Thomas BC, Rumilla KM, Thomas CS, Heckman MG, et al. A clinical scoring system to identify patients with sebaceous neoplasms at risk for the Muir-Torre variant of Lynch syndrome. Genet Med. 2014 Sep;16(9):711-6.

16 Carethers JM, Stoffel EM. Lynch syndrome and Lynch syndrome mimics: the growing complex landscape of hereditary colon cancer. World J Gastroenterol. 2015 Aug;21(31): 9253-61. 
17 Jessup MJ, Goldberg RM, Asare EA, et al. Colon and Rectum. In: Amin MB, Edge SB, editors. AJCC Cancer Staging Manual. 8th ed. Basel: Springer; 2017. pp. 251-74.

18 John AM, Schwartz RA. Muir-Torre syndrome (MTS): an update and approach to diagnosis and management. J Am Acad Dermatol. 2016 Mar;74(3):558-66.

19 Conway MR, Graue GF, Pelayes D, et al. Conjunctival Carcinoma. In: Amin MB, Edge SB, editors. AJCC Cancer Staging Manual. 8th ed. Basel: Springer; 2017. pp. 787-93.

20 Jakobiec FA. Sebaceous adenoma of the eyelid and visceral malignancy. Am J Ophthalmol. 1974 Dec;78(6):952-60.

21 Page EH, Assaad DM. Morpheic plaque of the lower eyelid. Sebaceous carcinoma of the eyelid. Arch Dermatol. 1987 May;123(5):654-5.

22 Tillawi I, Katz R, Pellettiere EV. Solitary tumors of meibomian gland origin and Torre's syndrome. Am J Ophthalmol. 1987 Aug; 104(2):179-82.

23 Jakobiec FA, Zimmerman LE, La Piana F, Hornblass A, Breffeilh RA, Lackey JK. Unusual eyelid tumors with sebaceous differentiation in the Muir-Torre syndrome. Rapid clinical regrowth and frank squamous transformation after biopsy. Ophthalmology. 1988 Nov;95(11):1543-8.

24 Stockl FA, Dolmetsch AM, Codere F, Burnier MN Jr. Sebaceous carcinoma of the eyelid in an immunocompromised patient with MuirTorre syndrome. Can J Ophthalmol. 1995 Oct;30(6):324-6.

25 Spraul CW, Lang GE, Grossniklaus HE, Lang GK. Metastatic adenocarcinoma to the retina in a patient with Muir-Torre syndrome. Am J Ophthalmol. 1995 Aug;120(2):248-50.

26 Entius MM, Keller JJ, Drillenburg P, Kuypers KC, Giardiello FM, Offerhaus GJ. Microsatellite instability and expression of hMLH-1 and hMSH-2 in sebaceous gland carcinomas as markers for Muir-Torre syndrome. Clin Cancer Res. 2000 May;6(5):1784-9.

27 Popnikolov NK, Gatalica Z, Colome-Grimmer MI, Sánchez RL. Loss of mismatch repair proteins in sebaceous gland tumors. J Cutan Pathol. 2003 Mar;30(3):178-84.

28 Tay E, Schofield JB, Rowell NP, Jones CA. Ophthalmic presentation of the Muir Torre syndrome. Ophthal Plast Reconstr Surg. 2003 Sep;19(5):402-4.
29 Rishi K, Font RL. Sebaceous gland tumors of the eyelids and conjunctiva in the Muir-Torre syndrome: a clinicopathologic study of five cases and literature review. Ophthal Plast Reconstr Surg. 2004 Jan;20(1):31-6.

30 Bassetto F, Baraziol R, Sottosanti MV, Scarpa C, Montesco M. Biological behavior of the sebaceous carcinoma of the head. Dermatol Surg. 2004 Mar;30(3):472-6.

31 Goldberg M, Rummelt C, Foja S, Holbach LM, Ballhausen WG. Different genetic pathways in the development of periocular sebaceous gland carcinomas in presumptive Muir-Torre syndrome patients. Hum Mutat. 2006 Feb;27(2):155-62.

32 Demirci H, Nelson CC, Shields CL, Eagle RC Jr, Shields JA. Eyelid sebaceous carcinoma associated with Muir-Torre syndrome in two cases. Ophthal Plast Reconstr Surg. 2007 JanFeb;23(1):77-9.

33 Tohya T, Ogura T, Nishi K, Nishi H, Kuriwaki K. Muir-Torre syndrome associated with endometrial carcinoma. Int J Clin Oncol. 2008 Dec;13(6):559-61.

34 Newman BD, Maher JF, Subauste JS, Uwaifo GI, Bigler SA, Koch CA. Clustering of sebaceous gland carcinoma, papillary thyroid carcinoma and breast cancer in a woman as a new cancer susceptibility disorder: a case report. J Med Case Reports. 2009 Jul;3(1):6905.

35 Agiannidis C, Pana ZD, Molyva D, Kalokasidis K, Mixiou M. Metachronous occurrence of colorectal cancer in a muir-torre syndrome patient presenting with recurrent sebaceous adenoma of the eyelid: case report and updated review of the literature. J Cutan Med Surg. 2012 Nov-Dec;16(6):394-9.

36 Rajan Kd A, Burris C, Iliff N, Grant M, Eshleman JR, Eberhart CG. DNA mismatch repair defects and microsatellite instability status in periocular sebaceous carcinoma. Am J Ophthalmol. 2014 Mar;157(3):640-7.e1.

37 Ishiguro Y, Homma S, Yoshida T, Ohno Y, Ichikawa N, Kawamura $\mathrm{H}$, et al. Usefulness of $\mathrm{PET} / \mathrm{CT}$ for early detection of internal malignancies in patients with Muir-Torre syndrome: report of two cases. Surg Case Rep. 2017 Dec;3(1):71.
38 Font RL, Rishi K. Sebaceous gland adenoma of the tarsal conjunctiva in a patient with Muir-Torre syndrome. Ophthalmology. 2003 Sep;110(9):1833-6.

39 Lai TF, Huilgol SC, Selva D, James CL. Eyelid sebaceous carcinoma masquerading as in situ squamous cell carcinoma. Dermatol Surg. 2004 Feb;30(2 Pt 1):222-5.

40 Singh AD, Mudhar HS, Bhola R, Rundle PA, Rennie IG. Sebaceous adenoma of the eyelid in Muir-Torre syndrome. Arch Ophthalmol. 2005 Apr;123(4):562-5.

41 Ponti G, Losi L, Pedroni M, Lucci-Cordisco E, Di Gregorio C, Pellacani G, et al. Value of MLH1 and MSH2 mutations in the appearance of Muir-Torre syndrome phenotype in HNPCC patients presenting sebaceous gland tumors or keratoacanthomas. J Invest Dermatol. 2006 Oct;126(10):2302-7.

42 Kamisasanuki T, Uchino E, Fukushima J, Yoshikawa H, Ishibashi T, Sakamoto T. A case of Muir-Torre syndrome with multiple cancers of bilateral eyelids and breast. Korean J Ophthalmol. 2013 Jun;27(3):204-7.

43 Gauthier AS, Campolmi N, Tumahai P, Kantelip B, Delbosc B. Sebaceous carcinoma of the eyelid and Muir-Torre syndrome. JAMA Ophthalmol. 2014 Aug;132(8):1025-8.

44 Mencía-Gutiérrez E, Gutiérrez-Díaz E, Santos-Briz A, Madero-García S. Sebaceous gland carcinoma of the eyelid and palpebral conjunctiva in a patient with Muir-Torre syndrome. Br J Ophthalmol. 2000 Nov;84(11): 1325-6.

45 Wong CY, Tang RK, Lam RF, Io IY, Yuen CY, Ho JW, et al. Muir-torre syndrome in a middle-aged Chinese patient with sebaceous carcinoma of the eyelid. Semin Ophthalmol. 2015;30(5-6):420-2.

46 Makrygiannis G, Vahdani K, Giasin O. MuirTorre Syndrome Masquerading as Chalazion. Ophthal Plast Reconstr Surg. 2015 Sep-Oct; 31(5):422-3.

47 Marcoval J, Talavera-Belmonte A, FornonsServent R, Bauer-Alonso A, Penín RM, Servitje $\mathrm{O}$. Cutaneous sebaceous tumours and Lynch syndrome: long-term follow-up of 60 patients. Clin Exp Dermatol. 2019 Jul;44(5): 506-511. 